\title{
Repetitive transcranial magnetic stimulation affects behavior by biasing endogenous cortical oscillations
}

\author{
Massihullah Hamidi ${ }^{1,2}{ }^{*}$, Heleen A. Slagter ${ }^{3}$, Giulio Tononi ${ }^{4}$ and Bradley R. Postle ${ }^{4,5}$ \\ Medical Scientist Training Program, University of Wisconsin - Madison, Madison, WI, USA \\ 2 Neuroscience Training Program, University of Wisconsin-Madison, Madison, WI, USA \\ 3 Waisman Laboratory for Brain Imaging and Behavior, University of Wisconsin - Madison, Madison, WI, USA \\ ${ }^{4}$ Department of Psychiatry, University of Wisconsin - Madison, Madison, WI, USA \\ ${ }^{5}$ Department of Psychology, University of Wisconsin-Madison, Madison, WI, USA
}

\section{Edited by:}

Rui M. Costa, Champalimaud Neuroscience Programme, Instituto Gulbenkian de Ciência, Portugal

\section{Reviewed by:}

Matias Palva, University of Helsinki, Finland

Ole Jensen, Donders Institute for Brain, Cognition and Behaviour, The Netherlands

\section{${ }^{*}$ Correspondence:}

Massihullah Hamidi, University of Wisconsin-Madison, 1202 West Johnson Street, Madison, WI 53706 , USA.

e-mail:mhamidi@wisc.edu
A governing assumption about repetitive transcranial magnetic stimulation (rTMS) has been that it interferes with task-related neuronal activity - in effect, by "injecting noise" into the brain -and thereby disrupts behavior. Recent reports of rTMS-produced behavioral enhancement, however, call this assumption into question. We investigated the neurophysiological effects of rTMS delivered during the delay period of a visual working memory task by simultaneously recording brain activity with electroencephalography (EEG). Subjects performed visual working memory for locations or for shapes, and in half the trials a $10-\mathrm{Hz}$ train of rTMS was delivered to the superior parietal lobule (SPL) or a control brain area. The wide range of individual differences in the effects of rTMS on task accuracy, from improvement to impairment, was predicted by individual differences in the effect of rTMS on power in the alpha-band of the EEG $(\sim 10 \mathrm{~Hz})$ : a decrease in alpha-band power corresponded to improved performance, whereas an increase in alpha-band power corresponded to the opposite. The EEG effect was localized to cortical sources encompassing the frontal eye fields and the intraparietal sulcus, and was specific to task (location, but not object memory) and to rTMS target (SPL, not control area). Furthermore, for the same task condition, rTMS-induced changes in cross-frequency phase synchrony between alpha- and gamma-band $(>40 \mathrm{~Hz})$ oscillations predicted changes in behavior. These results suggest that alpha-band oscillations play an active role cognitive processes and do not simply reflect absence of processing. Furthermore, this study shows that the complex effects of rTMS on behavior can result from biasing endogenous patterns of network-level oscillations.

Keywords: oscillations, alpha band, transcranial magnetic stimulation, rTMS, electroencephalography, working memory, spatial

\section{INTRODUCTION}

Although the storage of information in working memory has been studied for several decades, its underlying neurophysiological mechanisms remain unclear. Recently, several studies have utilized high-frequency $(>1 \mathrm{~Hz})$ repetitive transcranial magnetic stimulation (rTMS) during the delay-period of working memory tasks in order to explore the role of various brain areas implicated in storage (Hamidi et al., 2008; Luber et al., 2007; Postle et al., 2006). These studies have revealed that rTMS specifically to the parietal cortex alters working memory performance, suggesting a preferential role for the parietal cortex in storage. However, the direction of the behavioral effects varied, with both decrements (Postle et al., 2006) and improvements (Hamidi et al., 2008; Luber et al., 2007) reported. rTMS is often used with the assumption that it will produce electrical noise in the targeted region and will thereby disrupt task performance (Pascual-Leone et al., 2000; Walsh and Rushworth, 1999). There have been numerous demonstrations of such rTMS-induced "virtual lesions" that have produced theoretically interesting effects (e.g., Feredoes et al., 2006; Stewart et al., 2001). However, recent studies describing behavioral improvement with rTMS are not easily accommodated by a "virtual lesion" account and suggest that the physiological effects of rTMS are more complex.

One candidate alternative account is that rTMS, because it is delivered at regular intervals, interacts with endogenous neuronal oscillations in a manner that influences processing (Klimesch et al., 2003). Consistent with this idea, TMS pulses have been shown to affect oscillatory activity across many different frequency bands (Brignani et al., 2008; Fuggetta et al., 2008; Thut et al., 2003). There is also evidence of a role for neuronal oscillations in the storage of information in working memory (e.g., Jokisch and Jensen, 2007; Leiberg et al., 2006; Raghavachari et al., 2006). Thus, it is conceivable that the behavioral changes observed with rTMS in previous studies of working memory were due to an effect on task-related oscillatory activity.

We examined the effect of rTMS on working memory storage by recording the electroencephalogram (EEG) while simultaneously delivering $10-\mathrm{Hz}$ rTMS during the delay period of a delayed recognition working memory task. In addition to the fact that this stimulation frequency is commonly used in rTMS studies of cognition (e.g., Hamidi et al., 2009; Preston et al., in press; Rosenthal et al., 2008), $10 \mathrm{~Hz}$ was chosen because we previously observed a 
rTMS-induced improvement in working memory function with 10-Hz rTMS (Hamidi et al., 2008). We hypothesized that $10-\mathrm{Hz}$ rTMS, because it lies at the center of the alpha band $(8.5-14 \mathrm{~Hz})$, will interact with endogenous working memory-related alpha-band processes (e.g., Jensen et al., 2002; Michels et al., 2008). Additionally, because recent studies have emphasized the role of gamma-band $(>40 \mathrm{~Hz})$ activity in working memory (e.g., Jensen et al., 2007; Kaiser et al., 2009), we analyzed rTMS-induced changes in gammaband power, as well as cross-frequency phase relationships between alpha- and gamma-band oscillations (Palva et al., 2005).rTMS was applied to two brain areas, the superior parietal lobule (SPL) and a control brain area, the area representing the leg in the primary somatosensory cortex (S1), while subjects performed two different working memory tasks: one requiring memory of locations (spatial memory), the other requiring memory of shapes (object memory). Because the SPL is an area that is known to be involved in processing of spatial stimuli and spatial working memory (e.g., Schluppeck et al., 2006; Srimal and Curtis, 2008; Ungerleider et al., 1983), we expected a task-specific effect of SPL rTMS during location memory trials. The other three task conditions in our multifactorial design, during which we targeted a control brain area and/or used a ventral visual stream-dependent task (i.e., object memory) served as controls, in that each featured rTMS of a taskirrelevant brain area.

\section{MATERIALS AND METHODS SUBJECTS}

Sixteen young adults ( 12 male, mean age $=22.5$; $\mathrm{SD}=3.8$ ) were recruited from the University of Wisconsin community. Subjects did not have any psychiatric or neurological conditions, as determined by a psychiatrist or clinical psychologist who administered a structured psychiatric diagnostic interview (Mini-International Neuropsychiatric Interview, Sheehan et al., 1998) and mood assessment (Hamilton Depression Rating Scale, Hamilton, 1960). All subjects participating in this study provided informed consent and the protocol was approved by the University of Wisconsin Institutional Review Board.

\section{BEHAVIORAL TASK}

Subjects performed a delayed-recognition working memory task. Half the trials, randomly distributed, required memory for spatial locations, while the other half of trials required memory for shapes (Figure 1). Both trial types started with a 1-s display of instructions, indicating whether the upcoming trial required them to remember the identities (SHAPE) or the locations (LOCATION) of the subsequently presented shapes. After this instruction display, there was a 1-s fixation period, followed by the sequential presentation of four targets, abstract shapes (Arnoult and Attneave, 1956), of approximately $2^{\circ}$ of visual angle, presented for $1 \mathrm{~s}$ each. The target stimuli were followed by a luminance mask (100 ms), a 3-s delay period, and, finally, a probe stimulus (3 s). In location memory trials, the probe consisted of a white circle of $2^{\circ}$ of visual angle and subjects were required to determine, with a yes/no button press, whether the location of the probe matched that of any of the four targets. In the object memory trials, a shape was presented at the center of the screen and subjects had to make a decision as to whether the probe shape matched the shape of any one of the four targets (once again, with a yes/no button press). In both trial types the probe was presented for $3 \mathrm{~s}$ and subjects were limited to $3 \mathrm{~s}$ to make their response. To minimize eye movement artifacts in the EEG recordings, a fixation cross was presented for the duration of the task. Object and location trials were randomly interleaved. All trials were separated by an intertrial interval of $5 \mathrm{~s}$. In half the trials, randomly distributed, a 3-s, 10-Hz rTMS train was applied to the target brain area with the onset of the delay period. Accuracy and reaction time (RT) of the responses were recorded.

In order to assess whether the effect of $10-\mathrm{Hz}$ rTMS on behavior is dependent on endogenous alpha-band activity (as suggested by Klimesch et al., 2003), prior to the working memory task, we also recorded each subject's EEG, in three 1-min sessions while the subject had his/her eyes-closed and maintained a wakeful resting state. This allowed us to calculate their "natural" peak individual alpha-band frequency (IAF; Klimesch et al., 1990).

\section{rTMS}

rTMS was applied to the SPL and, as a control, S1 (c.f. Hamidi et al., 2008; Postle et al., 2006). Inclusion of a cortical control allowed us to account for any nonspecific effects of rTMS, such as scalp sensations and auditory noise, as well as any general physiological effects that rTMS may have on the cortex (Hamidi et al., in press). Both areas were identified based on individual anatomy

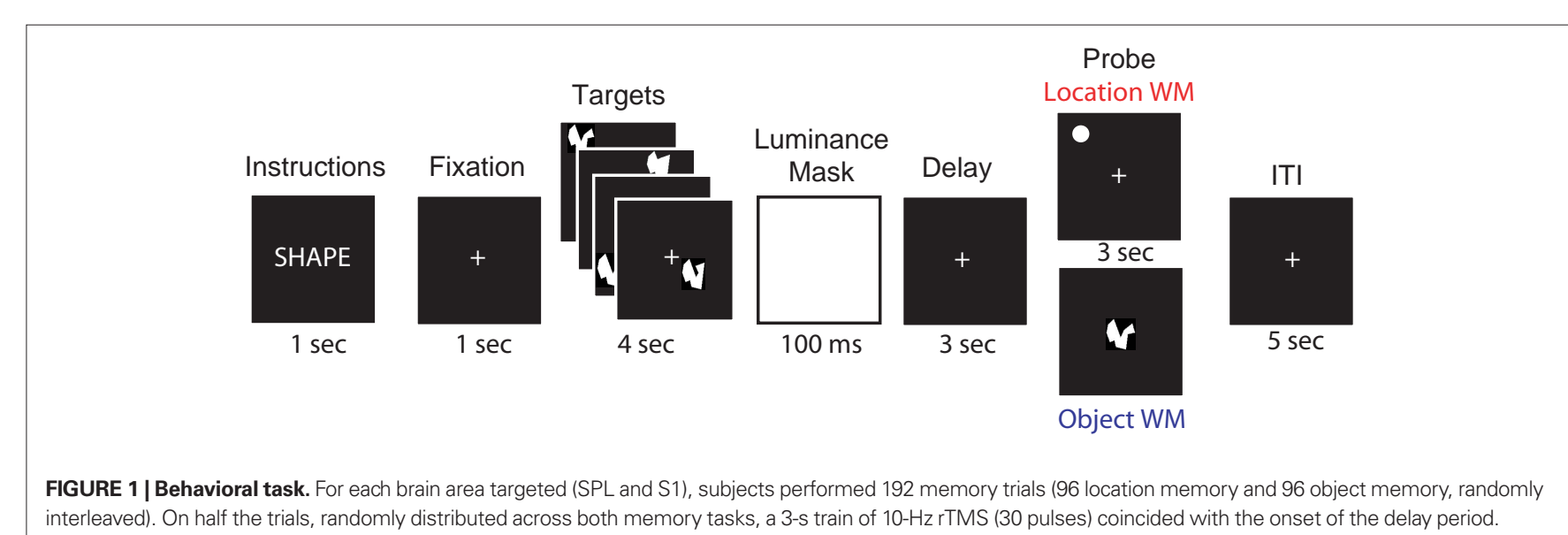


from whole-brain anatomical MRIs that were obtained for each subject prior to the study (GE Signa VH/I, 256 sagittal slices, $0.5 \mathrm{~mm} \times 0.5 \mathrm{~mm} \times 0.8 \mathrm{~mm}$ ). The SPL was identified as the area dorsal and medial to the intraparietal sulcus and posterior to the postcentral sulcus [Brodmann Area (BA) 7]. The S1 was identified as the area immediately posterior to the central sulcus, close to the midline. An infrared-based frameless stereotaxy system was used to accurately target each brain area with the TMS coil (eXimia Navigated Brain Stimulation, Nexstim, Helsinki, Finland). Because, in our previous study (Hamidi et al., 2008), there was no significant difference in the effect of left versus right SPL rTMS on behavior, for all subjects, rTMS was applied to the left hemisphere.

In rTMS $_{\text {present }}$ trials, a $10-\mathrm{Hz}$ rTMS train $[110 \%$ of motor threshold, corrected for scalp-to-cortex distance (Stokes et al., 2005)] was applied during the entire 3 -s delay period (30 pulses). TMS was delivered with a Magstim Standard Rapid magnetic stimulator fit with a 70-mm figure-8 stimulating coil (Magstim, Whitland, UK) placed against the scalp. Because the presence of rTMS was randomized, the inter-train interval varied. The stimulation parameters were well within the established safety limits (Wasserman, 1998).

\section{EEG RECORDINGS}

EEG was recorded with a 60-channel carbon cap and TMScompatible amplifier (Nexstim, Helsinki, Finland). This amplifier is designed to avoid saturation by the TMS pulse by employing a sample-and-hold circuit that keeps the output of the amplifier constant from $100 \mu$ s pre- to 2 ms post-stimulus (Virtanen et al., 1999). To further reduce residual TMS-related artifacts, the impedance at each electrode was kept below $3 \mathrm{k} \Omega$ (c.f. Massimini et al., 2005). The right mastoid was used as the reference and eye movements were recorded using two electrodes placed near the eyes. Data were acquired at a sampling rate of $1450 \mathrm{~Hz}$ with 16-bit resolution.

\section{DATA PREPROCESSING}

Data were processed offline using the EEGlab toolbox (version 5.03 and 6.01b, Delorme and Makeig, 2004) running in a MATLAB environment (Mathworks, Natick, MA, USA). The data were first down-sampled to $500 \mathrm{~Hz}$ (after application of a low-pass antialiasing filter) and then band-pass filtered between 0.1 and $500 \mathrm{~Hz}$. Following this, the data were cleaned of large movement-related artifacts and channels with excessive noise were reinterpolated using spherical spline interpolation (Perrin et al., 1989). Residual TMS-related electrical artifacts were identified and removed using independent component analysis (see Hamidi et al., in press, for a detailed description of the method). Before further analysis, the data were rereferenced to the average of all 60 electrodes.

\section{SPECTRAL ANALYSIS}

Delay-period oscillatory changes in power were measured by determining the event-related spectral perturbation (ERSP, Makeig, 1993 ) with each behavioral condition. ERSPs were computed using a moving Hanning-windowed wavelet with three cycles for the lowest frequency $(4 \mathrm{~Hz})$ increasing linearly to 30 cycles for the highest frequency analyzed $(80 \mathrm{~Hz})$. All ERSP analyses were performed over a time period from $500 \mathrm{~ms}$ prior to the onset of the delay period to $500 \mathrm{~ms}$ after the presentation of the probe stimulus (4-s epoch).
Mean delay-period ERSP was calculated separately for each subject and experimental condition (SPL/location memory; SPL/object memory; S1/location memory; S1/object memory). Responses were normalized for each subject by subtracting the calculated mean ERSP from that of a 2-s period within the middle of the intertrial interval for that subject. The effect of rTMS on ERSP was calculated by subtracting the mean ERSP during the $\mathrm{rTMS}_{\text {absent }}$ trials from the mean ERSP during the rTMS ${ }_{\text {present }}$ trials for each task condition.

IAF was determined by calculating the "center of gravity" frequency as described by Klimesch et al. (1990). This method takes into account individual differences in shape and distribution of the alpha-band peak.

\section{SOURCE LOCALIZATION}

Estimates of the cortical location of the rTMS-induced oscillatory changes were calculated using the FieldTrip toolbox ${ }^{1}$ and a linear beamforming technique (Gross et al., 2001; Van Veen et al., 1997). Source estimation was performed with a three-compartment model based on a standardized MRI of the brain (Oostenveld et al., 2001). The leadfield matrix was determined by using the boundary element model for each point in a regular 5-mm grid. At each point the source estimate was obtained with a spatial filter using the leadfield for the point and the cross-spectral density matrix from the data at each frequency of interest. The calculated transform was used to produce source estimates of the individual spectral information for each task condition. Normalized rTMS effect at each grid point was calculated for each subject using the following formula: rTMS effect $=\left(\mathrm{rTMS}_{\text {present }}-\mathrm{rTMS}_{\text {absent }}\right) / \mathrm{rTMS}_{\text {absent }}$. Source estimates were performed on the mean spectral power over the time and frequency ranges that showed significant correlations with behavior at the scalp level (see Figures 5 and 6).

\section{CORRELATION BETWEEN SPECTRAL POWER AND BEHAVIOR}

To determine if the rTMS-induced change in power is related to the rTMS-induced change in behavior, linear correlations between these two measures were calculated for each electrode across subjects. To assess for non-linear monotonic relationships, we also used non-parametric (Spearman's rho) measures of correlation. The results were similar for both measures, thus we only present linear correlations. Significance of the correlations was calculated by comparing the correlation coefficient to the normalized $(t)$ distribution of correlation coefficients from a population for which the correlation coefficient is 0 . For all time-frequency analyses, because working memory maintenance-related effects must be sustained over time, a temporal significance criterion was imposed, such that an effect had to be significant at $p<0.05$ continuously for at least one time-domain standard deviation of the wavelet used for analysis at the frequency of interest (i.e., for $10 \mathrm{~Hz}$, at least $94.9 \mathrm{~ms}$ of continuous time points must have a $p<0.05$; c.f., Slagter et al., 2009). The cortical locations of these correlations were estimated by correlating the rTMS-induced change in power at each voxel in the source solution with change in behavior across subjects. The source estimates were corrected for multiple comparisons by determining the minimum number of contiguous voxels required to reduce the probability of false positives to $p<0.01$ using Monte

${ }^{1}$ http://www.ru.nl/fcdonders/fieldtrip 
Carlo simulations as implemented by the AFNI software package (Cox, 1996; Ward, 2000). Based on this analysis, we only considered the source estimates of the correlations as significant if at least nine contiguous voxels showed a significant correlation at $p<0.05$. Using a similar method, we determined that with scalp topography, to account for multiple comparisons, an area showing significant correlations must encompass at least three contiguous channels.

\section{CROSS-FREOUENCY PHASE ANALYSES}

Recent evidence suggests that a critical function of neuronal oscillations may occur through phase interactions (Palva and Palva, 2007; Palva et al., 2005). Because gamma-band activity has been previously linked with storage of information in working memory (Jensen et al., 2007; Kaiser et al., 2009), we therefore analyzed the effect of rTMS on cross-frequency phase synchronization between alpha- and gamma-band oscillations and examined its relation to task performance. Cross-frequency phase-locking factor (PLF) was calculated as described by Palva et al. (2005). Briefly, the phases of oscillations at $f_{1}$ and $f_{2}$ were obtained by convolving the delay period signal ( $+500 \mathrm{~ms}$ on either end of the period) with a Morlet wavelet and calculating PLF based on the degree to which their phase difference was not uniformly distributed. PLF $=N^{-1} \Sigma z_{\text {, }}$, where $z_{i}$ describes complex valued measurement of the dependence between the two phases (Sinkkonen et al., 1995). $f_{1}$ and $f_{2}$ were chosen such that $n f_{1}=m f_{2}$ (Tass et al., 1998). In this study, we limited our analysis to the relationship between alpha- and gamma-band oscillations: $n=1(10 \mathrm{~Hz})$ and $m=4-6(40-60 \mathrm{~Hz})$. PLF calculations were performed over the second half of the delay period (which showed significant, task-dependent effects of rTMS on alpha-band power; see Figure 5). The effect of rTMS was determined by subtracting PLF during rTMS ${ }_{\text {present }}$ trials from that of rTMS $_{\text {absent }}$ trials. Correlations of this effect with behavior were calculated as described above.

\section{RESULTS}

\section{BEHAVIORAL EFFECTS}

Behavioral performance of one subject was deemed to be an extreme outlier (effect of rTMS was $>2$ standard deviations from the mean for both accuracy and RT) and data from this subject was thus excluded from all analyses. For the remaining subjects, on aggregate, for both target sites, rTMS had the selective effect of increasing accuracy on the location task, but not the object task (Figure 2). The effects of rTMS of these two regions differed, however, in the relation between rTMS effect on EEG and its effect on behavior.

\section{rTMS EFFECTS ON OSCILLATORY POWER}

With and without rTMS, the delay period was associated with a sustained increase in alpha-band power (Figure 3A), more so with object memory than location memory $\left(p<10^{-5}\right)$, a finding that has been observed in previous studies (e.g., Jokisch and Jensen, 2007). On aggregate, there were no significant changes in power with rTMS (Figures 3B,C). At the individual level, in all task conditions, rTMS produced large, sustained changes in delay-period alpha-band power, with some subjects showing an increase, and others showing a decrease in power. These individual differences in the effect of TMS on alpha-band power predicted its effect on behavior: On location memory trials, with SPL rTMS, there was a negative correlation between the effect of rTMS on accuracy and its effect on mean delay period alpha-band power (Figure 4). The sources of this effect were localized to three regions, a posterior frontal region, which included the frontal eye fields, a posterior region that extended from the ventral intraparietal sulcus to lateral occipital regions, and a region encompassing the right hippocampus (Figure 5A). There was no such relation for EEG signal from these cortical sources with rTMS to S1 and/or during object memory. In contrast, for each of the three control conditions, we

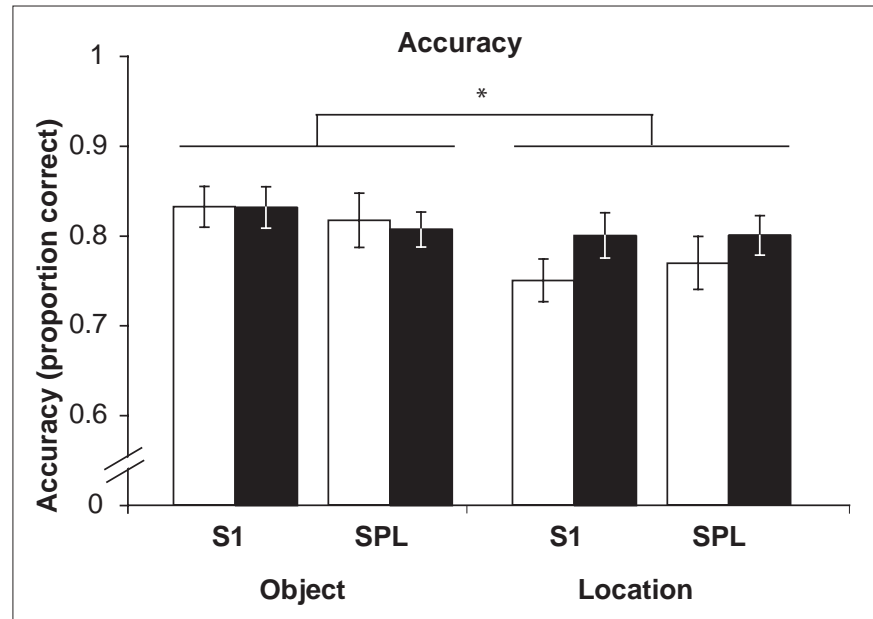

FIGURE 2 | Behavioral effect of rTMS. A three-way ANOVA (rTMS, brain area, memory task) on accuracy from revealed a main effect of memory task $[F(1,14)=5.22 ; p<0.05]$ and a significant $r T M S \times$ memory task interaction $[F(1,14)=4.72 ; p<0.05$, marked with an asterisk $]$. The main effect of memory task was driven by the fact that subjects had a higher accuracy in object memory trials compared to location memory trials [t(14) $=2.29 ; p<0.05]$. The rTMS $\times$ memory task interaction was due to an increase in accuracy with rTMS

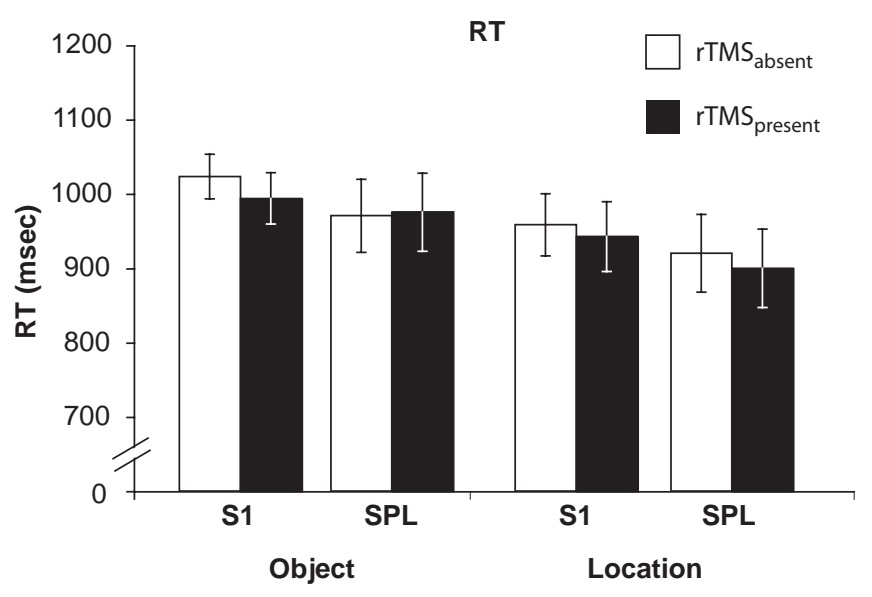

specifically during location working memory trials $[t(14)=2.17 ; p<0.05]$. All other effects and interactions were non-significant (all $F$-values $<1.47$ ). Three-way ANOVA (rTMS, brain area, memory task) on RT showed only a main effect of memory task $[F(1,14)=10.00 ; p<0.01]$. Subjects were faster at responding to location memory trials compared to object memory trials $[t(14)=3.16 ; p<0.01]$. There were no other significant main effects or interactions with $\mathrm{RT}$ (all F-values $<2.76$ ) 

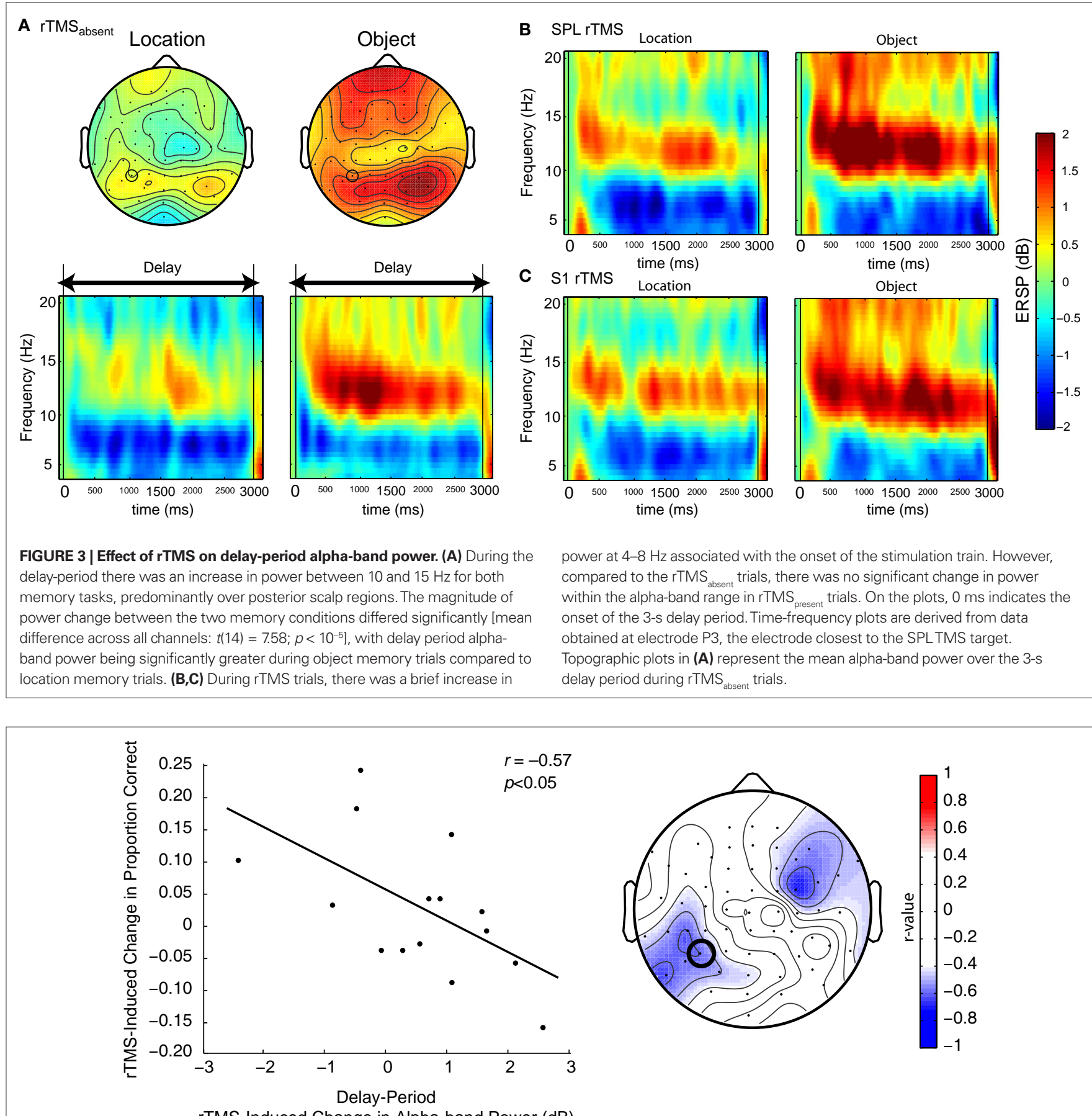

rTMS-Induced Change in Alpha-band Power (dB)

FIGURE 4 | Negative correlation between SPL rTMS-induced change in alpha-band power and change in accuracy during location working memory trials. Change in alpha-band power (averaged over $8.5-14 \mathrm{~Hz}$ ) was calculated for each subject as the mean difference in alpha-band power

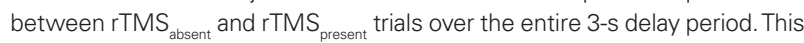
correlation was observed over a left, posterior cluster of channels, near the location of stimulation, and another right, frontal cluster of channels. For S1 rTMS and for both object memory conditions, there was a trend toward a positive relationship between rTMS-induced change in alpha-band power and change in accuracy (this trend became significant during the second half of the delay period). Data shown is from the electrode immediately below the TMS coil (electrode P3, circled). observed a positive correlation between the effect of rTMS on accuracy and its effect on alpha-band power (Figures 5B-D). These correlations significantly differed from that of SPL rTMS during location memory $\left(p\right.$-values $\left.<10^{-4}\right)$. The source estimates of these correlations varied, but in general were located in posterior parietal and bilateral occipital cortices (see Figure 5 for details). For all task conditions, the correlations were stronger during the second half of the delay period. 


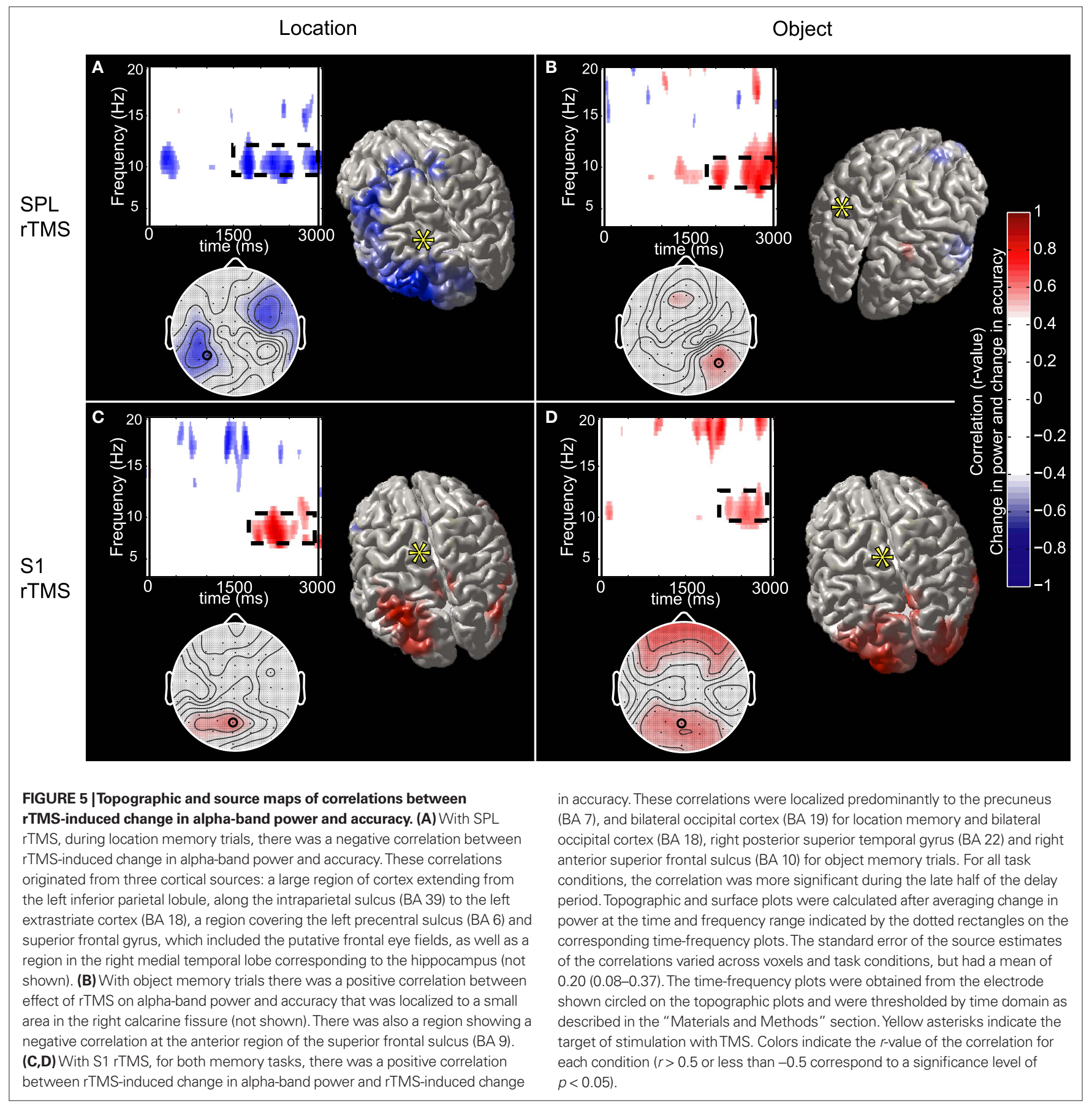

For three task conditions (SPL/location memory, SPL/object memory and S1/object memory), there was a positive correlation between rTMS-induced change in power between 14 and $16 \mathrm{~Hz}$ and RT (with an increase in power corresponding to slower responses). Additionally, for two control conditions (SPL/object memory and S1/location memory) there was a significant negative correlation between change in RT and change in power at approximately 7-10 Hz. The sources of these correlations were localized to several areas for each task condition without any regular pattern (details are presented in Figure $\mathbf{6}$ and Table 1).

Although on an individual level, with all task conditions, rTMS had variable effects on oscillations of other frequencies [theta(4-8 Hz), beta- $(15-30 \mathrm{~Hz})$, and gamma-bands], there were no consistent effects or any significant correlations between the effect of rTMS at these frequency bands and its effect on behavior.

\section{CROSS-FREOUENCY PHASE SYNCHRONY}

rTMS had no significant group-level effect on phase synchrony between alpha- and gamma-band oscillations for either object or location memory trials. However, with SPL rTMS, two clusters of 


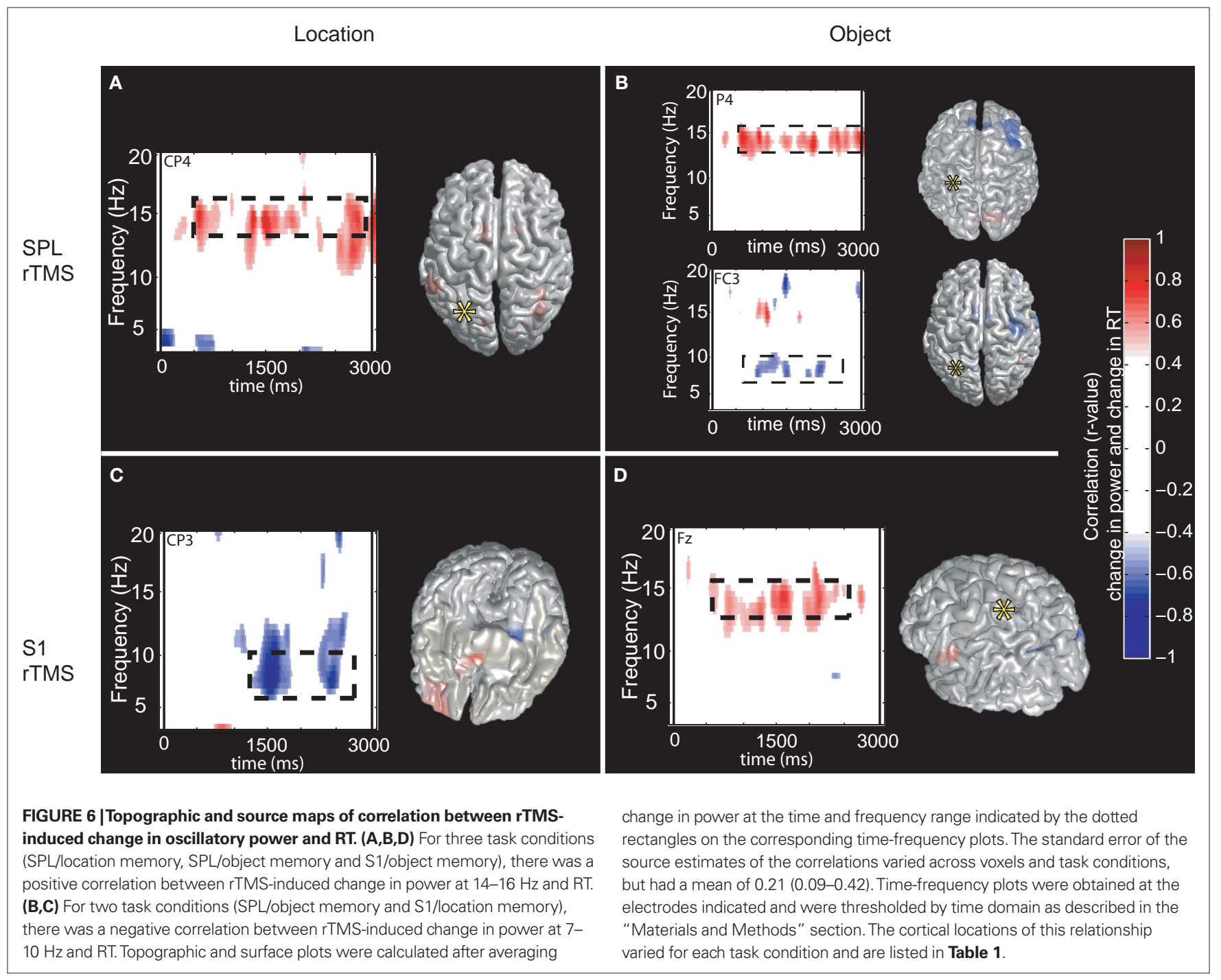

Table 1 | Cortical sources of correlations between rTMS-induced change in oscillatory power and rTMS-induced change in RT. For each task condition, correlations were calculated by determining the mean change in power over the time and frequency ranges indicated by the dotted rectangles in Figure 6 .

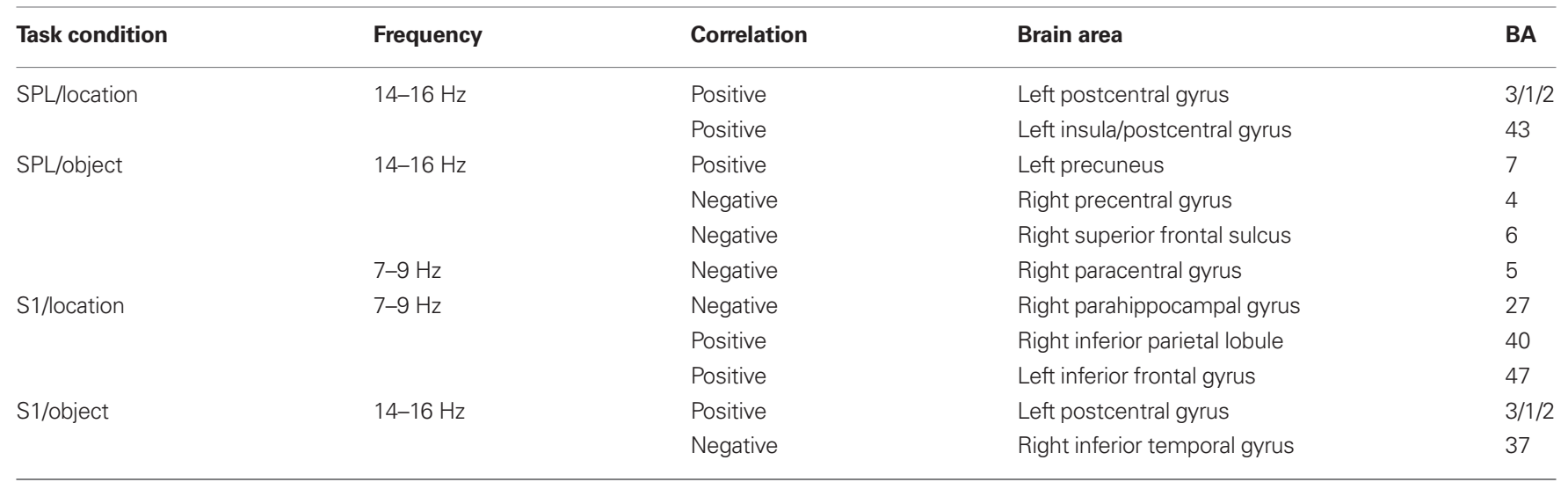

posterior electrodes (one in each hemisphere) evinced a relationship between PLF and accuracy, such that an increase in alpha: gamma phase synchrony was associated with improved behavioral performance during location trials, and with impaired behavioral performance during object trials (Figure 7). For S1 rTMS, we observed the opposite pattern, for location memory, the posterior electrodes trended toward a negative correlation, whereas for object memory they trended toward a positive correlation (although the 


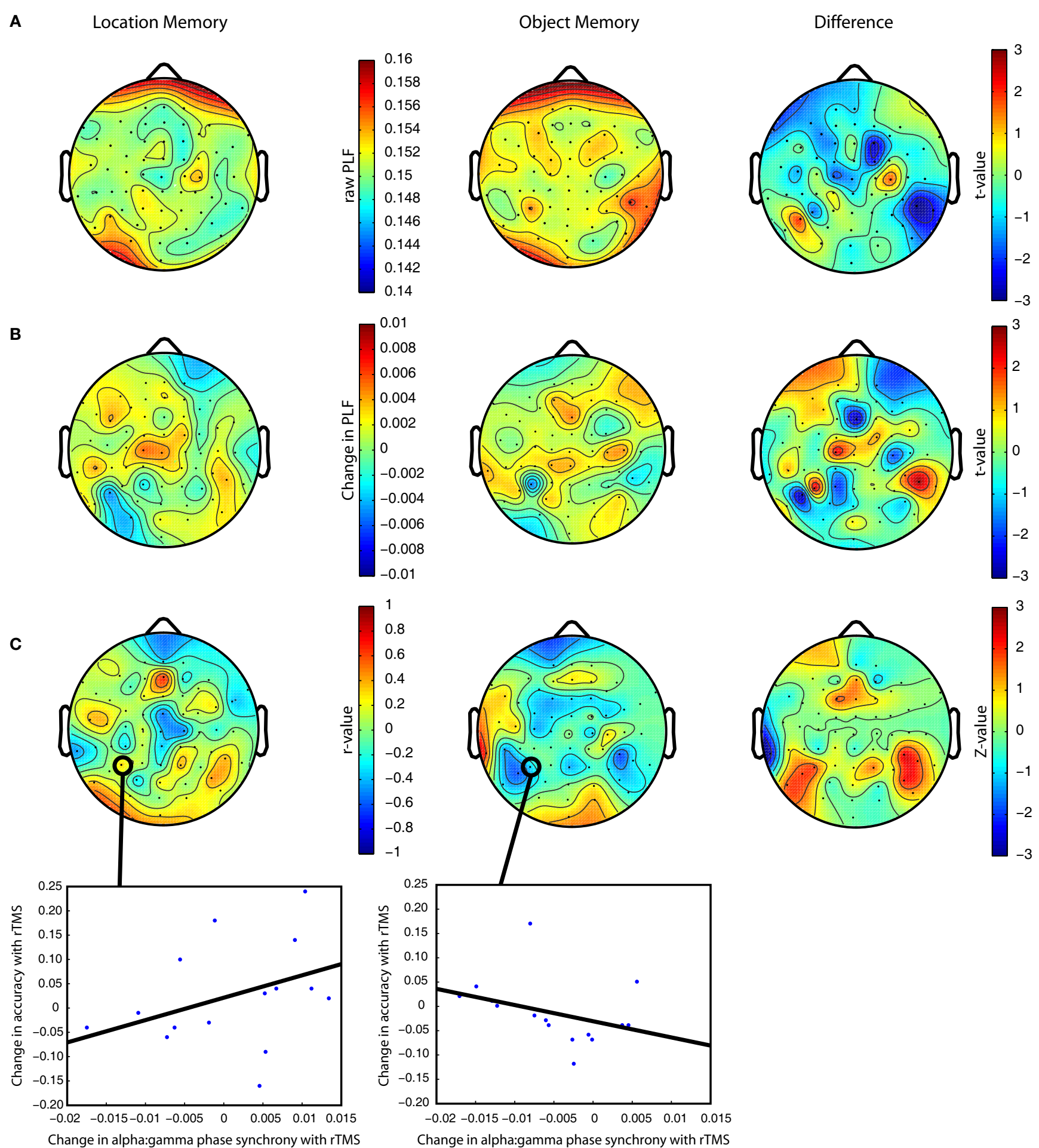

FIGURE 7 | (A) Raw alpha:gamma phase synchrony for location and object memory in TMMS $_{\text {absent }}$ trials. Pairwise analysis reveals a greater alpha:gamma synchronization in the right posterior electrodes during object memory trials. (B) Change in alpha:gamma phase synchrony with SPL rTMS. (C) Correlation between SPL rTMS-induced change in alpha:gamma phase synchrony and rTMS-induced change in accuracy $(r>0.50$ corresponds to $p<0.05)$. Scatter plots show this relationship for electrode P3 (nearest the location of stimulation). The difference between the correlations in object versus spatial memory trials was significant at right posterior electrodes $(Z=1.83-2.34$ for electrodes CP6, TP8 and $P 6 ; p=0.02-0.06)$ and marginally so for the left posterior electrodes $(Z=1.75-1.87$ for electrodes P7, P5 and P3; $p=0.06-0.08$ ). For S1 rTMS, the difference between the correlations for the two task conditions did not reach significance (there were no clusters of channels with $Z>2$ ). All plots are derived from the mean change in PLF during the second half of the delay period. All plots are based on phase synchrony between 10 and $40 \mathrm{~Hz}$ oscillations. Analysis of synchrony between 10 and 50 and 10 and $60 \mathrm{~Hz}$ reveals qualitatively similar results. 
difference between the two did not reach significance). The effects of rTMS on alpha:gamma phase synchrony across subjects were independent of its effects on alpha-band power (no significant relationship between the two; $r=0.14$ ).

\section{RESTING OSCILLATORY ACTIVITY AND rTMS EFFECT}

Because the behavioral effect of rTMS has been previously shown to be dependent on the relationship between frequency of stimulation and endogenous oscillatory activity as indicated by IAF (Klimesch et al., 2003), it is possible that the variability in the effect of rTMS in this study is due to differences in peak IAF across subjects. Thus, we evaluated the relation between peak IAF (measured in a separate rest session) and change in behavior with rTMS. With the experimental task condition there was a trend toward a positive linear relationship $(r=0.41 ; p=0.11)$ and a marginally significant quadratic relationship $(r=0.45 ; p=0.09)$ between peak IAF and rTMS-induced change in accuracy. These trends suggest that the effects of 10-Hz SPL rTMS on behavior may relate, at least in part, to an individual's peak alpha frequency. With all other task conditions we found no evidence for a significant relationship between peak IAF and change in accuracy (all $r$-values $<0.29$ ). Additionally, with all task conditions there were no significant relationships between peak IAF and rTMS-induced change in RT (all $r$-values <0.16). There was no significant relationship between each subject's peak IAF and the effect of rTMS on alpha:gamma phase synchrony for any task condition (all $r$-values $<0.37$ ).

\section{DISCUSSION}

The current study used EEG to examine the neural correlates of the behavioral effects of rTMS. We found that $10-\mathrm{Hz}$ rTMS-related changes in power in the alpha-band of the EEG selectively predicted corresponding changes in task accuracy, with a decrease in alpha-band power corresponding to improved performance, and an increase in alpha-band power corresponding to decreased performance. This effect was specific to task (spatial, but not object memory) and to rTMS target (SPL, not control area) and was localized to cortical sources implicated in the short-term retention of spatial information. These novel results suggest that the control of network-level oscillations in the alpha-band contributes importantly to the storage of information in working memory. Furthermore, they show that the complex effects of rTMS on behavior can result from biasing endogenous patterns of network-level oscillations. Below we discuss these findings and their implications in more detail.

\section{SPL rTMS INFLUENCES LOCATION MEMORY PERFORMANCE}

Our study findings indicate that $10-\mathrm{Hz}$ rTMS to SPL selectively enhances spatial working memory performance, and they extend previous findings (Hamidi et al., 2008), by showing that rTMS had no effect on object working memory ${ }^{2}$. This specificity is not surprising, since many studies have shown that the SPL is active during storage and processing of spatial information (e.g., Schluppeck

${ }^{2}$ The improvement in location memory performance with rTMS of S1 was unexpected, and was not observed in our previous study with a much larger sample size (Hamidi et al., 2008). Whatever its explanation, it is unrelated to the tight linkage with oscillations in the alpha-band that we observed with rTMS of SPL. et al., 2006; Srimal and Curtis, 2008; Ungerleider and Mishkin, 1982) and that lesions to the SPL can selectively impair spatial working memory (e.g., Ferber and Danckert, 2006). Object memory and processing of object visual information, on the other hand, is thought to rely more on object processing areas of the ventral visual stream (e.g., Postle et al., 2003; Ranganath et al., 2004; Ungerleider and Mishkin, 1982).

Our data also suggest that rTMS may modulate behavior via an effect on neuronal oscillations. During location memory trials, the observed negative relationship between rTMS-induced change in alpha-band power and rTMS-induced change in accuracy indicate that $10-\mathrm{Hz}$ rTMS to the SPL may directly affect spatial memoryrelated alpha band-dependent processes. It is notable that this effect was not localized to the tissue directly under the rTMS coil, but rather to two other regions in the network responsible for the storage and control of spatial information. Additionally, the effect did not occur at exactly $10 \mathrm{~Hz}$, suggesting that rTMS modulates endogenous oscillatory activity, rather than imposing its own rhythm. The direction of the observed correlation suggests that, in the dorsal visual stream, alpha-band activity may interfere with or inhibit storage of spatial information. Of course, the precision of cortical localization of EEG data is limited. Thus, one cannot draw definite conclusions about the neural generators of the scalprecorded activity. Nonetheless, the observed pattern in this study is generally consistent with the idea that one function of cortical alpha-band activity is inhibition of ongoing processing (Klimesch et al., 2007) and provides important support for the notion that rTMS may influence behavior by modulating endogenous oscillatory activity (Klimesch et al., 2003).

An intriguing question that arises from the results of this study is whether the relationship between rTMS and neuronal oscillations is dependent on the stimulation frequency used. As described above, it is unlikely that rTMS simply leads to entrainment of neural activity. Yet, previous studies have shown that altering stimulation frequency can dramatically affect the behavioral effects of rTMS (Klimesch et al., 2003; Luber et al., 2007). Thus, stimulation frequency is clearly important. Further research is required to determine why and a next step toward determining the mechanism behind rTMSinduced changes in endogenous oscillations would be to observe the effect of varying rTMS frequency on brain activity.

\section{FUNCTIONAL ROLE OF ALPHA-BAND ACTIVITY IN STORAGE}

Oscillations within the alpha band have been associated with decreased processing since the advent of EEG (Berger, 1929; Klimesch et al., 2007), and this idea has gained considerable support in recent years. For example, simultaneous EEG and 18-fluorodeoxyglucose positron emission tomography reveals a negative correlation between alpha-band EEG power and neuronal metabolism (Oakes et al., 2004). Similar results have been observed with simultaneous EEG and functional magnetic resonance imaging (e.g., Goldman et al., 2002; Laufs et al., 2003). Additionally, single pulses of TMS to the occipital cortex are more likely to induce phosphenes if delivered when spontaneous alpha-band power is low than when it is high (Romei et al., 2008). In the context of working memory, however, the role of alpha-band activity is less clear. Although EEG studies of verbal working memory have reported a positive relation between delay-period alpha-band power and 
working memory load (Jensen et al., 2002; Michels et al., 2008), a recent summary of findings from intracortical recordings reveals both positive and negative changes in "local" EEG depending on electrode location (Meltzer et al., 2008). A previous study of spatial versus object visual working memory with magnetoencephalography reported that parietal alpha-band power increased to a much greater extent during object memory trials compared to that of location memory trials (an effect that was replicated in this study), whereas parietal gamma-band activity (which is thought to signify local neuronal processing) was increased in location memory trials only. The observed increase in alpha-band power during object memory trials in parietal brain areas was taken to reflect inhibition of task-irrelevant (spatial) processing (Jokisch and Jensen, 2007). The opposite effects on behavior of rTMS-induced alpha-band changes with spatial versus object memory trials in this study verify this idea. In fact, for all three control conditions (in which rTMS was applied to task-irrelevant brain areas), a positive relationship between change in alpha-band power and change accuracy was observed. This observation provides further evidence that alphaband oscillations are performing a task-specific function. One possible explanation for the positive relationship observed in the control conditions may be that, by targeting task-irrelevant brain areas, rTMS-induced alpha-band oscillations decrease task-irrelevant processing and, thereby, improve memory performance. The current findings thus extend previous findings by showing that alpha activity may not only play a role in inhibiting task-irrelevant areas, but can also interfere with, or inhibit processing in taskrelevant brain areas.

Independent of the negative relationship between rTMSinduced changes in alpha-band power and accuracy, SPL rTMSinduced changes in alpha:gamma phase synchrony were positively related with task performance. This observation fits with the idea that phase and amplitude dynamics may underlie independent functions (Palva et al., 2005; Palva and Palva, 2007), as well as with models of cross-frequency nesting as a mechanism of information storage (e.g., Lisman and Idiart, 1995). Although it was not possible to determine the cortical sources of these cross-frequency effects, the observed pattern may suggest that rTMS-induced increases in alpha:gamma synchrony in posterior parietal brain regions result in improved location memory performance, whereas rTMS-induced increases in alpha:gamma synchrony in inferior and temporal brain regions result in improved object memory performance.

How alpha-band activity affects neuronal processing is unknown, although low frequency neuronal oscillations, including alpha, are thought to signify top-down attention-mediated control (Klimesch et al., 2007; Michels et al., 2008; von Stein et al., 2000). Yet, several groups have recently reported multiple independent alpha-band sources, suggesting that alpha-band activity may serve multiple functions (i.e., Bollimunta et al., 2008; Meltzer et al., 2008; Michels et al., 2008). For example, with intracortical recordings a recent study distinguished infragranular and supragranular alpha-band activity at different cortical areas and provided evidence for both alpha band-related top-down and bottom-up processing (Bollimunta et al., 2008). Thus, with respect to our data, it is conceivable that one alpha-band oscillator is performing an inhibitory function through amplitude modulation, whereas another is supporting memory via cross-frequency phase dynamics. How rTMS interacts with these processes to bring about the observed changes in brain activity requires further study. Nevertheless, together with prior work showing that the behavioral effects of rTMS are highly dependent on the frequency of stimulation (Klimesch et al., 2003; Luber et al., 2007), our data suggest that $10-\mathrm{Hz}$ stimulation likely directly interacts with endogenous neural processes across a wide range of frequencies. This novel finding suggests that oscillatory neuronal activity plays an active role in cognitive processing and is not a passive epiphenomenon (Klimesch et al., 2007).

\section{4-16 HZ OSCILLATIONS AND RT}

We also observed that rTMS-induced change in 14-16 Hz power correlated positively with RT, such that, an increase in $14-16 \mathrm{~Hz}$ power was associated with a slowing of RT. Interestingly, this frequency range falls within the frequency band associated with the mu motor rhythm and post-movement beta rhythm (Pfurtscheller and Neuper, 1997; Pfurtscheller et al., 1997). These rhythms predominate in brain areas involved in motor control and are maximal when movements are being inhibited (Chen et al., 1997; Hari et al., 2006). Thus, an intriguing possibility is that this rhythm also suppresses a motor preparation process resulting in a slowing of response.

\section{INDIVIDUAL DIFFERENCES}

The neurophysiological and behavioral effects of rTMS varied widely across subjects. The factors that contribute to these intersubject differences are unclear. We did not find any relations between gender or age and effect of rTMS (data not shown). There are several other factors that may explain the observed individual differences in rTMS-induced effects. One possibility concerns interindividual differences in the location of the peak IAF (Klimesch et al., 2003). In our experimental task condition, we found marginal evidence for a relationship between peak IAF and the effect of rTMS on behavior. Thus, the effect of rTMS may depend, at least in part, on the interaction between stimulation frequency and the "natural" frequency of the area targeted and/or the frequency of task-induced endogenous processes in the targeted area (Rosanova et al., 2009). Other possible explanations of variability in the effect of rTMS include differences in the orientation of the coil relative to the underlying anatomy (Bonato et al., 2006), differences in functional connectivity or use of alternative behavioral strategies during task performance.

Another explanation for the variable effects of rTMS across subjects may be that to a certain extent, the brain activity induced by rTMS facilitates cognitive processing, but above a certain level, the effect of rTMS becomes detrimental. In other words, the effect of rTMS on cognitive processing may follow an inverted-U pattern. Hence, for some subjects the peak of the facilitatory effects of rTMS may be higher than for others. Such an effect may be a result of stochastic resonance, such that, superimposition of a noisy signal by rTMS will facilitate detection of subthreshold oscillatory signals. However, after a certain point, the level of noise will overwhelm any underlying signal (Moss et al., 2004). This possibility can be tested by altering the stimulation intensity on an individual subject basis, and observing the effect of rTMS on brain activation and behavior. 
Although it is unclear why rTMS produces differential effects across subjects, this does not detract from our finding that rTMS-induces changes in working memory performance are related to its effect on alpha-band power. Importantly, the observed individual differences suggest that, perhaps, by tailoring the stimulation parameters for each subject, it may be possible to selectively inhibit or potentiate cognitive processing. Determining the factors underlying individual differences in rTMS effects will be important for refining the functional effects of rTMS in future studies and, potentially, for improving the clinical efficacy of rTMS.

\section{CONCLUSIONS}

The present findings reveal that rTMS may influence behavior by interacting with endogenous neuronal oscillations. Individual differences in rTMS-induced changes in behavior were predicted by individual differences in the effect of rTMS on power in the alpha-band of the EEG, an effect that was localized to a distributed

\section{REFERENCES}

Arnoult, M. D., and Attneave, F. (1956). The quantitative study of shape and pattern perception. Psychol. Bull. 53, 452-471.

Berger, H. (1929). Über das Elektroenkephalogramm des Menschen. Arch. Psychiatr. Nervenkr. 87, 527-570.

Bollimunta, A., Chen, Y., Schroeder, C. E., and Ding, M. (2008). Neuronal mechanisms of cortical alpha oscillations in awake-behaving Macaques. J. Neurosci. 28, 9976-9988.

Bonato, C., Miniussi, C., and Rossini, P.M. (2006). Transcranial magnetic stimulation and cortical evoked potentials: a TMS/EEG co-registration study. Clin. Neurophysiol. 117, 1699-1707.

Brignani, D., Manganotti, P., Rossini, P. M., and Miniussi, C. (2008). Modulation of cortical oscillatory activity during transcranial magnetic stimulation. Hum. Brain Mapp. 29, 603-612.

Chen, R., Classen, J., Gerloff, C., Celnik, P., Wassermann, E. M., Hallett, M., and Cohen, L. G. (1997). Depression of motor cortex excitability by low-frequency transcranial magnetic stimulation. Neurology 48, 1398-1403.

Cox, R.W. (1996).AFNI: software for analysis and visualization of functional magnetic resonance neuroimages. Comput. Biomed. Res. 29, 162-173.

Delorme, A., and Makeig, S. (2004). EEGLAB: an open source toolbox for analysis of single-trial EEG dynamics including independent component analysis. J. Neurosci. Methods 134, 9-21.

Ferber, S., and Danckert, J. (2006). Lost in space - the fate of memory representations for non-neglected stimuli. Neuropsychologia 44, 320-325.
Feredoes, E., Tononi, G., and Postle, B. R. (2006). Direct evidence for a prefrontal contribution to the control of proactive interference in verbal working memory. Proc. Natl. Acad. Sci. USA 103, 19530-19534.

Fuggetta, G., Pavone, E. F., Fiaschi, A., and Manganotti, P. (2008). Acute modulation of cortical oscillatory activities during short trains of high-frequency repetitive transcranial magnetic stimulation of the human motor cortex: a combined EEG and TMS study. Hum. Brain Mapp. 29, 1-13.

Goldman, R. I., Stern, J. M., Engel, J., and Cohen, M. S. (2002). Simultaneous EEG and fMRI of the alpha rhythm. Neuroreport 13, 2487-2492.

Gross, J., Kujala, J., Hamalainen, M., Timmermann, L., Schnitzler, A., and Salmelin, R. (2001). Dynamic imaging of coherence sources: studying neural interactions in the human brain. Proc. Natl. Acad. Sci. USA 98, 694-699.

Hamidi, M., Slagter, H.A., Tononi, G., and Postle, B. R. (in press). Brain responses evoked by high-frequency repetitive transcranial magnetic stimulation: an event-related potential study. Brain Stimulat.

Hamidi, M., Tononi, G., and Postle, B. R. (2008). Evaluating frontal and parietal contributions to spatial working memory with repetitive transcranial magnetic stimulation. Brain Res. 1230, 202-210.

Hamidi, M., Tononi, G., and Postle, B. R. (2009). Evaluating the role of prefrontal and parietal cortices in memoryguided response with repetitive transcranial magnetic stimulation. Neuropsychologia 47, 295-302.

Hamilton, M. (1960). A rating scale for depression. J. Neurol. Neurosurg. Psychiatr. 23, 56-62.

network implicated in spatial cognition. These results demonstrate that rTMS can have complex effects on neuronal oscillations with variable effects across subjects. They illustrate that the behavioral effects of rTMS are not necessarily due to induction of a "virtual lesion". In addition, in line with previous work, our results provide strong evidence that there are multiple, distinct alpha-band sources that independently support cognitive processing and, furthermore, that amplitude and phase dynamics both play active roles in cognition.

\section{ACKNOWLEDGEMENTS}

We would like to thank Lawrence Greischar, Alex Shackman and Michael Kruepke for their expert help and technical assistance. This work also benefited from discussions with Jeffrey Johnson, Marcello Massimini and Mario Rosanova. This study was supported by grants MH078705 (Massihullah Hamidi) and MH064498 (Bradley R. Postle) of the National Institute of Mental Health and by NARSAD (Giulio Tononi).

Hari, R., Neuper, C., and Klimesch, W. (2006).Action-perception connection and the cortical mu rhythm. Prog. Brain Res. 159, 253-260.

Jensen, O., Gelfand, J., Kounios, J., and Lisman, J. E. (2002). Oscillations in the alpha band $(9-12 \mathrm{~Hz})$ increase with memory load during retention in a short-term memory task. Cereb. Cortex 12, 877-882.

Jensen, O., Kaiser, J., and Lachaux, J.-P (2007). Human gamma-frequency oscillations associated with attention and memory. Trends Neurosci. 30, 317-324.

Jokisch, D., and Jensen, O. (2007). Modulation of gamma and alpha activity during a working memory task engaging the dorsal or ventra stream. J. Neurosci. 27, 3244-3251.

Kaiser, J., Rahm, B., and Lutzenberger, W. (2009). Temporal dynamics of stimulus-specific gamma-band activity components during auditory short-term memory. Neuroimage 44, 257-264.

Klimesch, W., Sauseng, P., and Gerloff, C. (2003). Enhancing cognitive performance with repetitive transcranial magnetic stimulation at human individual alpha frequency. Eur. J. Neurosci. 17, 1129-1133.

Klimesch, W., Sauseng, P., and Hanslmayr, S. (2007). EEG alpha oscillations: the inhibition-timing hypothesis. Brain Res. Rev. 53, 63-88.

Klimesch, W., Schimke, H., Ladurner, G., and Pfurtscheller, G. (1990). Alpha frequency and memory performance. J. Psychophysiol. 4, 381-390.

Laufs, H., Kleinschmidt, A., Beyerle, A. Eger, E., Salek-Haddadi, A., Preibisch, C., and Krakow, K. (2003). EEG-correlated fMRI of human alpha activity. Neuroimage 19, 1463.
Leiberg, S., Lutzenberger, W., and Kaiser, J. (2006). Effects of memory load on cortical oscillatory activity during auditory pattern working memory. Brain Res. 1120, 131-140.

Lisman, J., and Idiart, M. A. P. (1995). Storage of $7 \pm 2$ short-term memories in oscillatory subcycles. Science 267, 1512-1515.

Luber, B., Kinnunen, L. H., Rakitin, B. C., Ellsasser, R., Stern, Y., and Lisanby, S. H. (2007). Facilitation of performance in a working memory task with rTMS stimulation of the precuneus: Frequency- and time-dependent effects. Brain Res. 1128, 120-129.

Makeig, S. (1993). Auditory event-related dynamics of the EEG spectrum and effects of exposure to tones. Electroencephalogr. Clin. Neurophysiol. 86, 283-293.

Massimini, M., Ferrarelli, F., Huber, R. Esser, S. K., Singh, H., and Tononi, G. (2005). Breakdown of cortical effective connectivity during sleep. Science 309, 2228-2232.

Meltzer, J. A., Zaveri, H. P. Goncharova, I. I., Distasio, M. M., Papademetris, X., Spencer, S. S. Spencer, D. D., and Constable, R. T. (2008). Effects of working memory load on oscillatory power in human intracranial EEG. Cereb. Cortex 18, 1843-1855.

Michels, L., Moazami-Goudarzi, M., Jeanmonod, D., and Sarnthein, J. (2008). EEG alpha distinguishes between cuneal and precuneal activation in working memory. Neuroimage 40, 1296-1310.

Moss, F., Ward, L. M., and Sannita, W. G. (2004). Stochastic resonance and sensory information processing: a tutorial and review of application. Clin. Neurophysiol. 115, 267-281. 
Oakes, T. R., Pizzagalli, D. A., Hendrick, A. M., Horras, K. A., Larson, C. L., Abercrombie, H. C., Schaefer, S. M., Koger, J. V., and Davidson, R. J. (2004). Functional coupling of simultaneous electrical and metabolic activity in the human brain. Hum. Brain Mapp. 21, 257-270.

Oostenveld, R., Praamstra, P., Stegeman, D. F., and van Oosterom, A. (2001). Overlap of attention and movement-related activity in lateralized event-related brain potentials. Clin. Neurophysiol. 112, 477-484.

Palva, J. M., Palva, S., and Kaila, K. (2005). Phase synchrony among neuronal oscillations in the human cortex. J. Neurosci. 25, 3962-3972.

Palva, S., and Palva, J. M. (2007). New vistas for $\alpha$-frequency band oscillations. Trends Neurosci. 30, 150-158.

Pascual-Leone, A., Walsh, V., and Rothwell, J. (2000). Transcranial magnetic stimulation in cognitive neuroscience - virtual lesion, chronometry, and functional connectivity. Curr. Opin. Neurobiol. 10, 232-237.

Perrin, F., Pernier, J., Bertrand, O., and Echallier, J. F. (1989). Spherical splines for scalp potential and current density mapping. Electroencephalogr. Clin. Neurophysiol. 72, 184-187.

Pfurtscheller, G., and Neuper, C. (1997). Motor imagery activates primary sensorimotor area in humans. Neurosci. Lett. 239, 65-68.

Pfurtscheller, G., Stanc.K, A., and Edlinger, G. (1997). On the existence of different types of central beta rhythms below $30 \mathrm{~Hz}$. Electroencephalogr. Clin. Neurophysiol. 102, 316-325.

Postle, B. R., Druzgal, T. J., and D'Esposito, M. (2003). Seeking the neural substrates of working memory storage. Cortex 39, 927-946.

Postle, B. R., Ferrarelli, F., Hamidi, M., Feredoes, E., Massimini, M., Peterson, M., Alexander, A., and Tononi, G. (2006). Repetitive transcranial magnetic stimulation dissociates working memory manipulation from retention functions in the prefrontal, but not posterior parietal, cortex. J. Cogn. Neurosci. 18, 1712-1722.
Preston, G., Anderson, E., Silva, C., Goldberg, T., and Wassermann, E. (in press). Effects of $10 \mathrm{~Hz}$ rTMS on the neural efficiency of working memory. J. Cogn. Neurosci. 1-10.

Raghavachari, S., Lisman, J. E., Tully, M. Madsen, J. R., Bromfield, E. B., and Kahana, M. J. (2006). Theta oscillations in human cortex during a working-memory task: evidence for local generators. J. Neurophysiol. 95, 1630-1638.

Ranganath, C., Cohen, M. X., Dam, C. and D'Esposito, M. (2004). Inferior temporal, prefrontal, and hippocampal contributions to visual working memory maintenance and associative memory retrieval. J. Neurosci. 24, 3917-3925.

Romei, V., Brodbeck, V., Michel, C., Amedi, A., Pascual-Leone, A., and Thut, G. (2008). Spontaneous fluctuations in posterior $\alpha$-band EEG activity reflect variability in excitability of human visual areas. Cereb. Cortex 18, 2010-2018.

Rosanova, M., Casali, A., Bellina, V., Resta, F., Mariotti, M., and Massimini, M. (2009). Natural frequencies of human corticothalamic circuits. J. Neurosci. 29, 7679-7685.

Rosenthal, C. R., Hodgson, T. L., Husain, M., and Kennard, C. (2008). Supplementary eye field contributions to the execution of saccades to remembered target locations. Prog. Brain Res. 171, 419-423.

Schluppeck, D., Curtis, C. E., Glimcher, P. W., and Heeger, D. J. (2006). Sustained activity in topographic areas of human posterior parietal cortex during memory-guided saccades. J. Neurosci. 26, 5098-5108.

Sheehan, D. V., Lecrubier, Y., Sheehan, K. H., Amorim, P., Janavs, J., Weiller, E., Hergueta, T., Baker, R., and Dunbar, G.C. (1998). The Mini-International Neuropsychiatric Interview (M.I.N.I.): the development and validation of a structured diagnostic psychiatric interview for DSM-IV and ICD-10. J. Clin. Psychiatry 59, 22-33.

Sinkkonen, J., Tiitinen, H., and Naatanen, R. (1995). Gabor filters: an informative way of analysing event-related brain activity. J. Neurosci. Methods 56, 99-104.

Slagter, H. A., Lutz, A., Greischar, L. L., Nieuwenhuis, S., and Davidson, R. J. (2009). Theta phase synchrony and conscious target perception: impact of intensive mental training. J. Cogn. Neurosci. 21, 1536-1549.

Srimal,R., and Curtis, C.E. (2008).Persistent neural activity during the maintenance of spatial position in working memory. Neuroimage 39, 455-468.

Stewart, L., Walsh, V., Frith, U., and Rothwell, J. C. (2001). TMS produces two dissociable types of speech disruption. Neuroimage 13, 472-478.

Stokes, M. G., Chambers, C. D., Gould, I. C., Henderson, T. R., Janko, N. E., Allen, N. B., and Mattingley, J. B. (2005). A simple metric for scaling motor threshold based on scalp-cortex distance: application to studies using transcranial magnetic stimulation. J. Neurophysiol. 94 4520-4527.

Tass, P., Rosenblum, M. G., Weule, J., Kurths, J., Pikovsky, A., Volkmann, J., Schnitzler, A., and Freund, H.-J. (1998). Detection of n:m phase locking from noisy data: Application to magnetoencephalography. Phys. Rev. Lett. 81, 3291-3294.

Thut, G., Théoret, H., Pfennig, A., Ives, J., Kampmann, F., Northoff, G., and Pascual-Leone, A. (2003). Differential effects of low-frequency rTMS at the occipital pole on visual-induced alpha desynchronization and visualevoked potentials. Neuroimage 18 334-347.

Ungerleider, L. G., Galkin, T. W., and Mishkin, M. (1983). Visuotopic organization of projections from striate cortex to inferior and lateral pulvinar in rhesus monkey. J. Comp. Neurol. 217, 137-157.

Ungerleider, L. G., and Mishkin, M. (1982). Two cortical visual systems In Analysis of Visual Behavior, D. J. Ingle, M. A. Goodale and R. J. W. Mansfield, eds (Cambridge, MA: MIT Press), pp. 549-586.

Van Veen, B. D., van Drongelen, M. Yuchtman, M., and Suzuki, A. (1997).
Localization of brain electrical activity via linearly constrained minimum variance spatial filtering. IEEE Trans. Biomed. Eng. 44, 867-880.

Virtanen, J., Ruohonen, J., Naatanen, R., and Ilmoniemi, R. J. (1999). Instrumentation for the measurement of electric brain responses to transcranial magnetic stimulation. Med. Biol. Eng. Comput. 37, 322-326.

von Stein, A., Chiang, C., and Konig, P. (2000). Top-down processing mediated by interareal synchronization. Proc. Natl. Acad. Sci. USA 97, 14748-14753.

Walsh, V., and Rushworth, M. (1999). A primer of magnetic stimulation as a tool for neuropsychology. Neuropsychologia 37, 125-135.

Ward, B. D. (2000). Simultaneous inference for fMRI data. http://afni.nimh. nih.gov/afni/doc/manual/AlphaSim.

Wasserman, E. (1998). Risk and safety of repetitive transcranial magnetic stimulation: report and suggested guidelines from the International Workshop on the Safety of Repetitive Transcranial Magnetic Stimulation, June 5-7, 1996. Electroencephalogr. Clin. Neurophysiol. 108, 1-16.

Conflict of Interest Statement: The authors declare that the research was conducted in the absence of any commercial or financial relationships that could be construed as a potential conflict of interest.

Received: 28 February 2009; paper pending published: 10 April 2009; accepted:09 June 2009; published online: 24 June 2009.

Citation: Hamidi M, Slagter HA, Tononi $G$ and Postle BR (2009) Repetitive transcranial magnetic stimulation affects behavior by biasing endogenous cortical oscillations. Front. Integr. Neurosci. (2009) 3:14. doi: 10.3389/neuro.07.014.2009

Copyright $@ 2009$ Hamidi, Slagter, Tononi and Postle. This is an open-access article subject to an exclusive license agreement between the authors and the Frontiers Research Foundation, which permits unrestricted use, distribution, and reproduction in any medium, provided the original authors and source are credited. 